\title{
A MINIMAL PAIR IN THE GENERIC DEGREES
}

\author{
DENIS R. HIRSCHFELDT
}

\begin{abstract}
We show that there is a minimal pair in the nonuniform generic degrees, and hence also in the uniform generic degrees. This fact contrasts with Igusa's result that there are no minimal pairs for relative generic computability, and answers a basic structural question mentioned in several papers in the area.
\end{abstract}

Generic computability is a notion of "almost everywhere computability" introduced by Kapovich, Myasnikov, Schupp, and Shpilrain [11. Beginning with the work of Jockusch and Schupp [9], it and the related notion of coarse computability have been studied from the computability-theoretic viewpoint by several authors. (See Jockusch and Schupp [10] for a survey. Coarse computability had actually been consider earlier by Terwijn [14.) Here, "almost everywhere" is defined in terms of (asymptotic) density. A set $A$ has density 1 if $\lim _{n} \frac{\mid A\lceil n \mid}{n}=1$, and has density 0 if its complement has density 1.

Definition 1. A generic description of a set $A$ is a partial function $f$ such that $\operatorname{dom} f$ has density 1 and $f(n)=A(n)$ whenever $f(n)$ is defined. A set is generically computable if it has a partial computable generic description.

A coarse description of a set $A$ is a set $C$ such that $\{n: C(n)=A(n)\}$ has density 1 . A set is coarsely computable if it has a computable coarse description.

Thus generic computability captures the idea of computing a set while allowing for a small number of errors of omission, while coarse computability captures the idea of computing a set while allowing for a small number of errors of commission. We can also consider notions that allow both kinds of errors, as was done by Astor, Hirschfeldt, and Jockusch [1].

We can of course relativize the above notions to an oracle. We can also use them to define notions of reducibility. For coarse reducibility, doing so is straightforward, though there are two natural versions. (The fact that these versions are different, as are the analogous ones for generic computability defined below, was shown by Dzhafarov and Igusa [4.)

Definition 2. We say that $A$ is nonuniformly coarsely reducible to $B$ if every coarse description of $A$ computes a coarse description of $B$. We say that $A$ is uniformly coarsely reducible to $B$ if there is a Turing functional $\Phi$ such that if $C$ is a coarse description of $B$, then $\Phi^{C}$ is a coarse description of $A$.

Generic descriptions are partial functions, so we cannot use them directly as oracles, but we can use their graphs, together with the notion of enumeration reducibility, which does not allow us to use negative information about an oracle.

Partially supported by grants DMS-1600543 and DMS-1854279 from the National Science Foundation. I thank Carl Jockusch for reading and commenting on a draft of the proof of the main result, and later a draft of the paper itself. 
Recall that an enumeration operator is a c.e. set $W$ of pairs $(F, k)$ with each $F$ finite. For an oracle $X$, let $W^{X}=\{k: \exists(F, k) \in W[F \subseteq X]\}$. Then $Y$ is enumeration reducible to $X$ if there is an enumeration operator $W$ such that $Y=W^{X}$. We identify a partial function with its graph, so for partial functions $f$ and $g$, we say that $f$ is enumeration reducible to $g$ if $\operatorname{graph}(f)$ is enumeration reducible to $\operatorname{graph}(g)$, and write $W^{g}$ for $W^{\operatorname{graph}(g)}$. We write $W^{X}[s]$ for $\{k: \exists(F, k) \in W[s][F \subseteq X]\}$. The use of an enumeration $k \in W^{X}[s]$ is the least $n$ such that $\exists(F, k) \in W[s][F \subseteq$ $X \uparrow n]$.

Definition 3. We say that $A$ is nonuniformly generically reducible to $B$ if for every generic description $f$ of $B$, there is a generic description of $A$ that is is enumeration reducible to $f$. We say that $A$ is uniformly generically reducible to $B$ if there is an enumeration operator $W$ such that if $f$ is a generic description of $B$, then $W^{f}$ is a generic description of $A$.

These reducibilities induce equivalence relations on $2^{\omega}$, from which degree structures arise as usual. For any degree structure with a least element $\mathbf{0}$, a minimal pair is a pair of degrees $\mathbf{a}, \mathbf{b}>\mathbf{0}$ such that if $\mathbf{c}<\mathbf{a}$ and $\mathbf{c}<\mathbf{b}$ then $\mathbf{c}=\mathbf{0}$. Most degree structures studied in computability theory have minimal pairs, and proving this fact is often one of the first structural results one establishes about such a structure. For the (nonuniform and uniform) coarse degrees, the existence of minimal pairs was proved by Hirschfeldt, Jockusch, Kuyper, and Schupp [6]. For the generic degrees, however, the following basic question had remained open.

Question 4 (Jockusch and Schupp [9]; Igusa [7; see also [5]). Are there minimal pairs in the (nonuniform or uniform) generic degrees?

An interesting aspect of this question is its relationship to relative generic computability. In the case of coarse computability, the aforementioned proof of the existence of minimal pairs follows from the following stronger result. (See e.g. [2] for more on notions of algorithmic randomness.)

Theorem 5 (Hirschfeldt, Jockusch, Kuyper, and Schupp [6]). If X is not coarsely computable and $Y$ is weakly 3 -random relative to $X$ then $X$ and $Y$ form a minimal pair for relative coarse computability. That is, if a set is coarsely computable relative both to $X$ and to $Y$, then it is coarsely computable.

In the generic case, however, the situation is quite different.

Theorem 6 (Igusa [7]). There are no minimal pairs for relative generic computability. That is, if $X$ and $Y$ are not computable, then there is a set that is not generically computable, but is generically computable relative both to $X$ and to $Y$.

Notice that this result does not imply that there are no minimal pairs for generic reducibility, because being generically computable relative both to $X$ and to $Y$ is a weaker condition than being generically reducible both to $X$ and to $Y$. For a set $A$ to have the latter property, not only do both $X$ and $Y$ have to enumerate generic descriptions of $A$, but so does every generic description of $X$ or of $Y$. Thus we have some extra power in trying to build a minimal pair for generic reducibility, which we will exploit below, relying particularly on the fact that enumeration reducibility cannot make use of information about inputs on which a partial computable function is undefined, and hence is monotonic, in the sense that for an enumeration operator $W$, if the partial function $g$ extends the partial function $f$, then any number enumerated into $W^{f}$ is also enumerated into $W^{g}$. 
On the other hand, Theorem [6] does show that the methods of [6], and the related ones of [1, are not available here. Nevertheless, in this paper we give a positive answer to Question 4 in both cases. (Notice that it is enough to consider the nonuniform case, since if the nonuniform generic degrees of two sets form a minimal pair, then so do their uniform generic degrees.) Despite solving a reasonably wellknown open problem, the proof is fairly short. It is inspired by the construction of a minimal pair of c.e. Turing degrees, but is a finite injury construction that relies on the monotonicity of enumeration operators.

We now give the proof, followed by a few comments on coarse computability, further work of Igusa [8], and the notions of dense and effective dense computability studied in [1].

Theorem 7. There is a minimal pair in the nonuniform generic degrees (and hence in the uniform generic degrees).

Proof. Let $R_{e}=\left\{n: 2^{e} \mid n \wedge 2^{e+1} \nmid n\right\}$. Let $W_{0}, W_{1}, \ldots$ be an effective listing of the enumeration operators.

We will build $\Delta_{2}^{0}$ sets $A_{0}$ and $A_{1}$, and the following functions defined from these sets: Let $f_{j, s}$ be the partial function defined by letting $f_{j, s}(n)=1$ if $n \notin A_{j}[s]$, and $f_{j, s}(n) \uparrow$ if $n \in A_{j}[s]$. Let $f_{j}$ be the partial function defined by letting $f_{j}(n)=1$ if $n \notin A_{j}$, and $f_{j}(n) \uparrow$ if $n \in A_{j}$.

We will build $A_{0}$ and $A_{1}$ to have the following properties.

(1) Each $A_{j} \cap R_{e}$ is finite, so $A_{j}$ is almost entirely contained in each $\bigcup_{e>k} R_{e}=$ $\left\{n>0: 2^{e+1} \mid n\right\}$, and hence has density 0 .

(2) If $\operatorname{dom} \Phi_{e} \cap R_{e}$ is infinite then $\operatorname{dom} \Phi_{e} \cap R_{e} \cap A_{j} \neq \emptyset$.

(3) For each $e_{0}, e_{1}$, and $s$, if $x \in W_{e_{0}}^{f_{0, s}}[s] \cap W_{e_{1}}^{f_{1, s}}[s]$, then $x \in W_{e_{j}}^{f_{j}}$ for some $j \leqslant 1$.

Assuming we have done so, if $n \in \operatorname{dom} \Phi_{e} \cap R_{e} \cap A_{j}$ then let $X_{j}(n) \neq \Phi_{e}(n)$, and let $X_{j}(n)=1$ for all other $n$. We claim that the generic degrees of $X_{0}$ and $X_{1}$ form a minimal pair

If $\operatorname{dom} \Phi_{e}$ has density 1 then $\operatorname{dom} \Phi_{e} \cap R_{e}$ is infinite, so by property (2), there is an $n \in \operatorname{dom} \Phi_{e} \cap R_{e} \cap A_{j}$, and hence $\Phi_{e}$ is not a generic description of $X_{j}$. Thus neither $X_{j}$ is generically computable.

By property (1) and the definition of $X_{j}$, each $f_{j}$ is a generic description of $X_{j}$, so if $Y$ is generically reducible to both $X_{0}$ and $X_{1}$, then there are $e_{0}$ and $e_{1}$ such that each $W_{e_{j}}^{f_{j}}$ is a generic description of $Y$. Let $\Psi$ be defined as follows. For each $n$, search for an $s$ and a $k \leqslant 1$ such that $\langle n, k\rangle \in W_{e_{0}, s}^{f_{0, s}}[s] \cap W_{e_{1}}^{f_{1, s}}[s]$. If one is found, then let $\Psi(n)=k$. By property (3), if $\Psi(n)=k$ then $\langle n, k\rangle \in W_{e_{j}}^{f_{j}}$ for some $j \leqslant 1$, which implies that $Y(n)=k$. There are density 1 many $n$ such that $\langle n, Y(n)\rangle \in W_{e_{0}}^{f_{0}} \cap W_{e_{1}}^{f_{1}}$. For any such $n$, there must be an $s$ such that $\langle n, Y(n)\rangle \in$ $W_{e_{0}}^{f_{0, s}}[s] \cap W_{e_{1}}^{f_{1, s}}[s]$, whence $\Psi(n)=Y(n)$. Thus $\Psi$ is a generic description of $Y$. But $\Psi$ is partial computable, so $Y$ is generically computable.

Thus the nonuniform generic degrees of $X_{0}$ and $X_{1}$ form a minimal pair, and hence so do their uniform generic degrees.

To explain the basic idea for building $A_{0}$ and $A_{1}$, consider requirements

$$
\mathcal{P}_{e, j}:\left|\operatorname{dom} \Phi_{e} \cap R_{e}\right|=\omega \Rightarrow \operatorname{dom} \Phi_{e} \cap R_{e} \cap A_{j} \neq \emptyset
$$


and

$$
\mathcal{N}_{e_{0}, e_{1}}: \forall s \forall x\left[x \in W_{e_{0}}^{f_{0, s}}[s] \cap W_{e_{1}}^{f_{1, s}}[s] \Rightarrow \exists j\left[x \in W_{e_{j}}^{f_{j}}\right]\right],
$$

arranged into a priority list as usual. (In fact, only the $\mathcal{P}$-requirements need to be assigned priorities.)

Let us consider the interaction of a requirement $\mathcal{N}_{e_{0}, e_{1}}$ with the requirements $\mathcal{P}_{e, j}$. (Different $\mathcal{N}$-requirements will not interact with each other.) Whenever we have $x \in W_{e_{0}}^{f_{0, s}}[s] \cap W_{e_{1}}^{f_{1, s}}[s]$, we want to preserve at least one of the computations that have led to the enumerations of $x$. Each $\mathcal{P}_{e, j}$ acts by waiting for a witness $n \in \operatorname{dom} \Phi_{e} \cap R_{e}$ to appear, and then putting $n$ into $A_{j}$. Doing so might destroy computations we are trying to preserve, but only on the $j$-side. We can now force all requirements weaker than $\mathcal{P}_{e, j}$ to choose witnesses beyond the uses of all computations we are trying to preserve, thus keeping the $(1-j)$-side from harm by such requirements. (Notice that it is not $\mathcal{N}_{e_{0}, e_{1}}$ that imposes this restraint, but $\mathcal{P}_{e, j}$, which is why the restraint imposed on a particular $\mathcal{P}$-requirement will be bounded, even as $\mathcal{N}_{e_{0}, e_{1}}$ has more and more computations it wants to preserve.)

But what if a requirement $\mathcal{P}_{e^{\prime}, 1-j}$ stronger than $\mathcal{P}_{e, j}$ wants to act, say at stage $t>s$ ? Then we must let it do so, which might destroy computations on the $(1-j)$ side. To compensate for that possibility, we remove the numbers we have put into $A_{j}$ for the sake of $\mathcal{P}_{e, j}$ (or any other requirement weaker than $\mathcal{P}_{e^{\prime}, 1-j}$ ). The key observation here is the following: this action does not necessarily restore $f_{j, t}$ to be the same as $f_{j, s}$, but assuming that $\mathcal{P}_{e^{\prime}, 1-j}$ is the strongest requirement to act since stage $s$, it ensures that $f_{j, t} \supseteq f_{j, s}$, which means that every element of $W_{e_{j}}^{f_{j, s}}[s]$ is also in $W_{e_{j}}^{f_{j, t}}[t]$.

We now turn to the formal construction of $A_{0}$ and $A_{1}$. For each $e, j$, we have a restraint $r(e, j)$, initially set to 0 . We adopt the common convention that a use defined at stage $s$ cannot be larger than $s$.

At stage $s$, let $\langle e, j\rangle<s$ be least such that $\operatorname{dom} \Phi_{e}[s] \cap R_{e} \cap A_{j}[s]=\emptyset$ and there is an $n \in \operatorname{dom} \Phi_{e}[s] \cap R_{e}$ that is larger than $r\left(e^{\prime}, j^{\prime}\right)$ for all $\left\langle e^{\prime}, j^{\prime}\right\rangle\langle\langle e, j\rangle$. (If there is no such pair $e, j$ then proceed to the next stage.) We say that $\mathcal{P}_{e, j}$ acts at stage $s$. Put $n$ into $A_{j}$. For each $\left\langle e^{\prime}, 1-j\right\rangle>\langle e, j\rangle$, remove every number put into $A_{1-j}$ at a previous stage at which $\mathcal{P}_{e^{\prime}, 1-j}$ acted. Let $r(e, j)=s$.

We now verify that this construction has the desired properties.

If a requirement puts a number into $A_{j}$ and that number is later removed at stage $s$, then any number put into $A_{j}$ by that same requirement at a later stage will be bigger than $s$. Since only $\mathcal{P}_{e, j}$ can ever put a number into $R_{e} \cap A_{j}$, and the $R_{e}$ 's are disjoint, each $A_{j}$ is $\Delta_{2}^{0}$ (in fact, d.c.e.).

Assume by induction that each $\mathcal{P}_{e^{\prime}, j^{\prime}}$ with $\left\langle e^{\prime}, j^{\prime}\right\rangle\langle\langle e, j\rangle$ stops acting, say by stage $t$. Then all the corresponding restraints $r\left(e^{\prime}, j^{\prime}\right)$ stop changing, so if $\operatorname{dom} \Phi_{e} \cap$ $R_{e}$ is infinite then either there is some $n \in \operatorname{dom} \Phi_{e}[t] \cap R_{e} \cap A_{j}[t]$ or $\mathcal{P}_{e, j}$ eventually gets to act after stage $t$ and put a number $n$ into dom $\Phi_{e} \cap R_{e} \cap A_{j}$. In either case, $n$ is never later removed from $A_{j}$, so property (2) holds. Furthermore, $\mathcal{P}_{e, j}$ acts at most once after stage $t$, so the induction can continue, and $\mathcal{P}_{e, j}$ puts at most finitely elements into $A_{j} \cap R_{e}$ after stage $t$ (in fact, at most one), so property (1) also holds.

Now fix $e_{0}, e_{1}$. Suppose that $x \in W_{e_{0}}^{f_{0, s}}[s] \cap W_{e_{1}}^{f_{1, s}}[s]$. Let $\langle e, j\rangle$ be the least pair such that $\mathcal{P}_{e, j}$ ever acts at a stage $t \geqslant s$. Then every element put into $A_{1-j}$ between stages $s$ and $t$ is removed from $A_{1-j}$ at stage $t$, so $f_{1-j, t} \supseteq f_{1-j, s}$, and 
hence $x \in W_{e_{1-j}}^{f_{1-j, t}}[t]$. By the definition of $r(e, j)$ at this stage, $f_{1-j}$ cannot change below the use of this enumeration after stage $t$, so $x \in W_{e_{1-j}}^{f_{1-j}}$. Thus property (3) is satisfied.

The sets $X_{j}$ built in the proof of Theorem 7 have density 1, which means that they are coarsely computable, and is also interesting in light of work of Igusa [8]: Astor, Hirschfeldt, and Jockusch 1 showed that the upper cone above any nontrivial (nonuniform or uniform) generic degree has measure 0, so a minimal generic degree would necessarily be half of a minimal pair. It is open whether there are minimal generic degrees, but Igusa [8] showed that the generic degree of a density- 1 set cannot be minimal. Interestingly, he also showed that if a uniform generic degree does not bound a nontrivial uniform generic degree containing a density- 1 set, then it is half of a minimal pair, but again it is not known whether such degrees exist.

It is also worth noting that the $X_{j}$ are $\Delta_{2}^{0}$. In fact, they are both co-d.c.e. (Notice that a c.e. density- 1 set is generically computable.) The case of Theorem 6 where both sets are $\Delta_{2}^{0}$ had been proved earlier by Downey, Jockusch, and Schupp 3 . By Theorem [5, the coarse degree of every set that is not coarsely computable is half of a minimal pair, and indeed forms minimal pairs with the coarse degrees of measure-1 many sets, but the following questions are open.

Open Question 8. Are there $\Delta_{2}^{0}$ sets whose (nonuniform or uniform) coarse degrees form a minimal pair?

The same question can be asked for the notion of dense computability introduced by Astor, Hirschfeldt, and Jockusch [1]. (In that paper, they showed that the resulting degree structures do have minimal pairs.)

Open Question 9. Is the (nonuniform or uniform) generic degree of every set that is not generically computable half of a minimal pair? What is the measure of the set of all $X \oplus Y$ such that the generic degrees of $X$ and $Y$ form a minimal pair? More generally, what can be said about the distribution of minimal pairs in the generic degrees?

Astor, Hirschfeldt, and Jockusch [1 also studied the following notion, which had been briefly considered much earlier by Meyer [13, and Lynch [12.

Definition 10. An effective dense description of a set $A$ is a (total) function $f: \omega \rightarrow\{0,1, \square\}$ such that $f^{-1}(\square)$ has density 0 and $f(n)=A(n)$ whenever $f(n) \in\{0,1\}$. A set is effectively densely computable if it has a computable effective dense description.

We say that $A$ is nonuniformly effectively densely reducible to $B$ if every effective dense description of $A$ computes an effective dense description of $B$. We say that $A$ is uniformly effectively densely reducible to $B$ if there is a Turing functional $\Phi$ such that if $f$ is an effective dense description of $B$, then $\Phi^{f}$ is an effective dense description of $A$.

These reducibilities lead to degree structures, for which the following question remains open. (It is also open whether there are minimal pairs for relative effective dense reducibility.)

Open Question 11 (Astor, Hirschfeldt, and Jockusch [1]). Are there minimal pairs in the (nonuniform or uniform) effective dense degrees? 
It does not seem that the proof of Theorem 7 can be adapted to this case in a straightforward way, because the key observation mentioned in the informal description of the construction in that proof does not apply here: Suppose that we make changes to the $j$-side of a pair of convergent computations, then find another such pair, and then again make changes to the $j$-side. If we later want to make changes to the $(1-j)$-side, we can no longer restore both computations on the $j$ side, because they might be based on different oracles. Because we are dealing with Turing functionals rather than enumeration operators, the only way to guarantee a return to a previous computation is to return to the exact original oracle (up to the relevant use). A proof along the lines of the usual construction of a minimal pair of c.e. Turing degrees, where we try to preserve at least one side of a pair of convergent computations up to their length of agreement (see e.g. 2, Section 2.14.2]), also seems problematic, because if a computation converges on both sides to 0 , say, and then the computation on one side disappears, when that computation converges again, it might converge to $\square$, which now allows the computation on the other side to change to 1 without creating a disagreement.

\section{REFERENCES}

[1] E. P. Astor, D. R. Hirschfeldt, and C. G. Jockusch, Jr., Dense computability, upper cones, and minimal pairs, Computability 8 (2019) 155-177.

[2] R. G. Downey and D. R. Hirschfeldt, Algorithmic Randomness and Complexity, Theory and Applications of Computability, Springer, New York, 2010.

[3] R. G. Downey, C. G. Jockusch, Jr., and P. E. Schupp, Asymptotic density and computably enumerable sets, J. Math. Log. 13 (2013) 1350005, 43 pp.

[4] D. D. Dzhafarov and G. Igusa, Notions of robust information coding, Computability 6 (2017) 105-124.

[5] D. R. Hirschfeldt, Some questions in computable mathematics, in Day, Fellows, Greenberg, Khoussainov, Melnikov, and Rosamond, eds., Computability and Complexity, Springer 2017, $22-55$.

[6] D. R. Hirschfeldt, C. G. Jockusch, Jr., R. Kuyper, and P. E. Schupp, Coarse reducibility and algorithmic randomness, J. Symbolic Logic 81 (2016) 1028-1046.

[7] G. Igusa, Nonexistence of minimal pairs for generic computation, J. Symbolic Logic 78 (2013) 511-522.

[8] G. Igusa, The generic degrees of density-1 sets, and a characterization of the hyperarithmetic reals, J. Symbolic Logic 80 (2015) 1290-1314.

[9] C. G. Jockusch, Jr. and P. E. Schupp, Generic computability, Turing degrees, and asymptotic density, J. London Math. Soc. 85 (2012) 472-490.

[10] C. G. Jockusch, Jr. and P. E. Schupp, Asymptotic density and the theory of computability : A partial survey, in Day, Fellows, Greenberg, Khoussainov, Melnikov, and Rosamond, eds., Computability and Complexity, Springer 2017, 501-520.

[11] I. Kapovich, A. Myasnikov, P. Schupp, and V. Shpilrain, Generic-case complexity, decision problems in group theory and random walks, J. Algebra 264 (2003) 665-694.

[12] N. Lynch, Approximations to the halting problem, J. Comput. System Sci. 9 (1974) 143-150.

[13] A. R. Meyer, An open problem on creative sets, Recursive Function Theory Newsletter 4 (1973) 15-16.

[14] S. A. Terwijn, Computability and Measure, PhD dissertation, University of Amsterdam, 1998.

Department of Mathematics, The University of Chicago

E-mail address: drh@math.uchicago.edu 